\title{
Accident Prevention Black-Box and Vehicle-to-Vehicle Communication Using Li-Fi and Wi-Fi Technologies
}

\author{
Aishwarya $\mathrm{I}^{\mathrm{a}, 1}$, Carolin $\mathrm{B}^{\mathrm{b}}$, ChalceEbenS $\mathrm{S}^{\mathrm{b}}$, Dr. P. Kannan ${ }^{\mathrm{c}}$ \\ ${ }^{a, b} U G$ Scholar, Dept of ECE, Panimalar Engineering College, Chennai, India \\ ${ }^{c}$ Head of Department, Dept of ECE, Panimalar Engineering College, Chennai, India
}

\begin{abstract}
The focus of the paper is Vehicle to Vehicle (V2V) communication system that uses a wireless communication technology using a LED transmitter and therefore the vehicle will transmit the information continuously to the opposite vehicle ahead it using Head light. The data is stored within the Secure Digital Card in comma separated value for future reference just in case of emergency at the equivalent time the data's are stored in the Cloud Server for Government Reference to locate the accident prone zones. The Light fidelity (Li-Fi) is that the newest technology within the field of wireless communication. As the number of users are increasing, the speed of knowledge transmission within the wireless network gets automatically decreased.
\end{abstract}

Keywords: Wireless-Fidelity (Wi-Fi), Light-Fidelity (Li-Fi), Light Emitting Diode (LED), Visible light communication (VLC), Stored Data, Accident Detection System, Black Box.

\section{Introduction}

The modern technology is always in a state of constant evolution, utilising all the resources available to make man's life a better place. Harald Hass, a professor from the university of Edinburgh, UK had come up with an idea of communication through light and termed it as Light-Fidelity' or 'Li-Fi', similar to the concept of wireless fidelity(which utilises radio waves to transmit data) but uses the visual light range to pass the information between the devices.Humans sometimes may make rash decisions under pressure resulting in an accident. Likely so, in highways at night. Practically, there can never be a $100 \%$ result and accidents occur due to an unforeseen cause. In this report, the focus is on improving the current vehicle-to-vehicle communication system and makes it more reliable. While taking in to account of the limitless potential of the visual light communication technology and integrating it with a Black box analysis system, it ismade to give a detailed report on severity of the accident and possibly giving aclue on how the accident had occurred, making the medical help be more preparedprovide effective treatment, thus saving lives.

\footnotetext{
${ }^{1}$ Aishwarya.I, UG Scholar, Dept of ECE, PanimalarEngineering College, Chennai, India. E-mail: aishwaryailango1703@gmail.com
} 


\section{Related works}

Nikhil Kumar, et al. [1] have proposed an accident detection model that works continuously whether accidents are detected or not and notifies the predetermined services selected in case accidents occur. The system classifies accidents so that the appropriate rescue operations can be taken. The disadvantage of the proposed system is that if an accident is occurred there is no data stored and available for future reference.Ali Hassan, et al. [2] have proposed an automatic accident detection system that uses an android app, vibration sensor, accelerometer, and Bluetooth module. The model relays alter SMS with precise location of the crash. The disadvantage of the model is that it cannot communicate with a nearby vehicle. Rahul George, et al. [3] have proposed a review on Li-Fi for Vehicle-to-Vehicle Communication. The journal proposes a model for vehicle-to-vehicle communication using Li-Fi technology. Li-Fi used essentially for its speed, High-density coverage, security, cost efficient and larger spectrum. The disadvantage of the paper is that it does not have a functioning model.Namrata H. Sane, et al. [4] have proposed a vehicle accident detection and tracking using GSM and GPS system. The system consists of an accident detection unit that is installed in the front and rear of the vehicle. The accident detection unit is integrated with Push on Switches, if an accident occurs then the Push on Switch senses the object and transmits a signal to interrupt pins of the microcontroller module. The system can be handled manually in case of minor accidents. The disadvantage ofthe proposed system is that it doesn't have an analysis module for after the occurrence of an accident.V Anupriya, et al. [5] have proposed an accident notification and collision avoidance system. The proposed system avoids accidents due to collisions between vehicles. It has an integrated smart rescue system in the corresponding vehicle that send the location of the spot in which the accident has occurred to the nearest hospital or clinic. And also the smart rescue system updates the health status of the patient and sends the status to the nearest hospital. The disadvantage of the proposed system is that it doesn't facilitate vehicle to vehicle communication. Hema Patel, et al. [6] have conducted Survey on Li-fi technology and its applications. The following paper emphasizes on the importance of $\mathrm{Li}$-fi, its working, application, which includes security, connectivity and navigation. The disadvantage is that the paper doesn't discuss the applications of Li-Fi in real time.

\section{Proposed System}

The proposed system contains v2v Communication using Li-Fi because Li-Fi is a fastest means of communication at the current period and Black-Box System is added to identify when, where and how the fault is occurred during accidents. Thereby making the system more trustworthy. The Fig. 1 is the block diagram of the transmitter module and Fig. 2 is the block diagram of the receiver module in setup. 


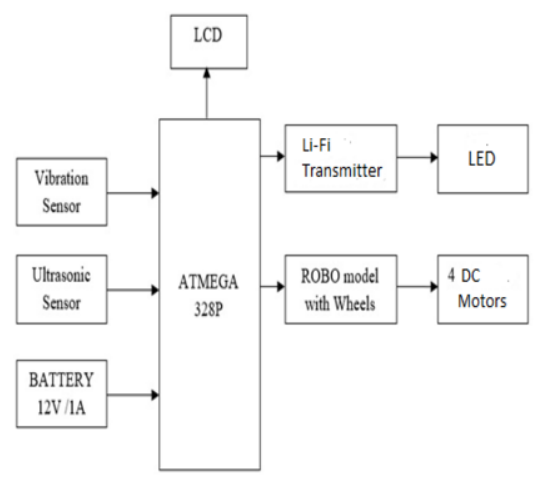

Figure 1.Transmittermodule

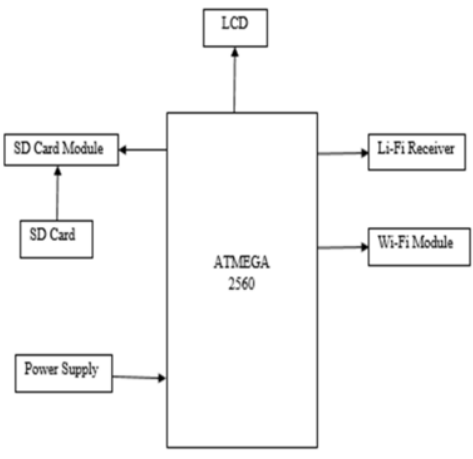

Figure 2.Receivermodule

\section{Requirements}

The hardware components used are Arduino MEGA 2560, Arduino ATMEGA 328P, LCD screen (16x2), Vibration Sensor, Ultrasonic Sensor, Piezoelectric Sensor, SD Card Module, Power Supply and Li-Fi transmitter and receiver.The cloud service used for this setup isThingSpeak (https://thingspeak.com/). The software used to program the instructions into the Arduino is ArduinoIDE 1.8.13.

\section{Working}

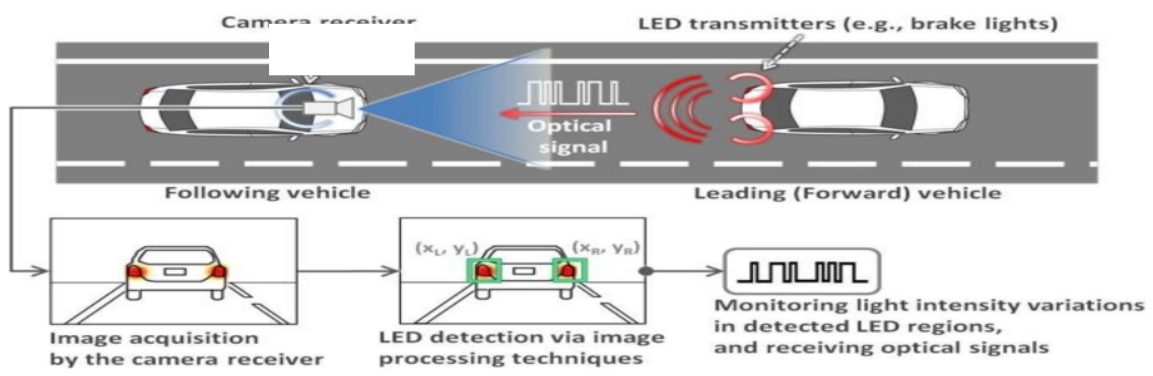

Figure 3. Working Module

\subsection{Working Description}

The above Fig. 3 is the working module. The goal of this setup is to relay 'alert messages' to the drivers in time, thereby preventing possible accidents. The messages are passed by the use of Visual Light Communication and wireless fidelity (Wi-Fi) system in the vehicles. The distance between the vehicle nearby and its speed is measured using Ultrasonic sensor. The microcontroller module controls the entire system and notifies the driver with an "alert message" when the nearby vehicle meets 
the line of sight. The black box system consists of a SD card module that stores information at regular intervals and stores it on the cloud. The stored information can be later retrieved in case accident has occurred. The black box system can be useful even if the vehicle-to-vehicle communication fails. The proposed system is very beneficial especially during the night time, where there is difficulty in vision and the lack of CCTV makes it hard to figure out the reason behind the accident occurred. To make it more effective black box concept is implemented here which stores the data in Wi-Fi and SD card which could be used if any mishap takes place and will play a major role during interrogation. The distance between the vehicles must be less than $30 \mathrm{~cm}$, so that the ultrasonic sensor detects and stops the vehicle automatically. Later the data is stored in the cloud and SD memory card for future reference. According to the German physicist Harald Hass, Li-Fi provides more speed (10megabits per second) data transmission by using visible light. In this, the speed of Li-Fi and Wi-Fi are compared and analyzed. The existing system has a transmitter and a receiver in each vehicle in both rear and front sides of the vehicle. Two scenarios will be studied in this paper. When the vehicle 1 is in the range of detection, a message will be sent through the transmitter which is placed in the rear lights to vehicle 2. A photodiode which is placed at the front of vehicle 2 to receive a notice of alert will be displayed in vehicle 2 using an LCD display. The message containing the information will be received by the photodiode in vehicle 2 and compared to vehicle 2 speeds. All the details of the road conditions from the first vehicle can be known only when the user is inside the car. The exact latitude \& longitude parameters of the vehicle can be known.

\section{Result and Discussion}

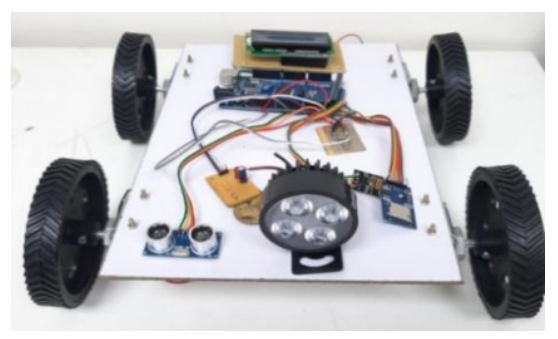

Figure 4. The top view of the Transmitter model.

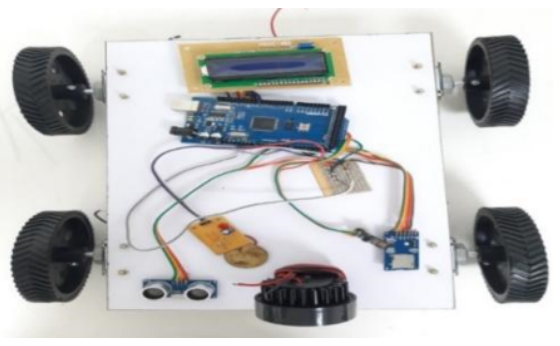

Figure 5. The front view of the Receiver model.

The above Fig. 4 is the top view of the model of Vehicle 1 and Fig. 5 is the front view of the model of Vehicle 1. Due to the extended communication range, this technique can provide certain information about other vehicles equipped with an equivalent technology. The system also helps to determine the location and time of the accident. 


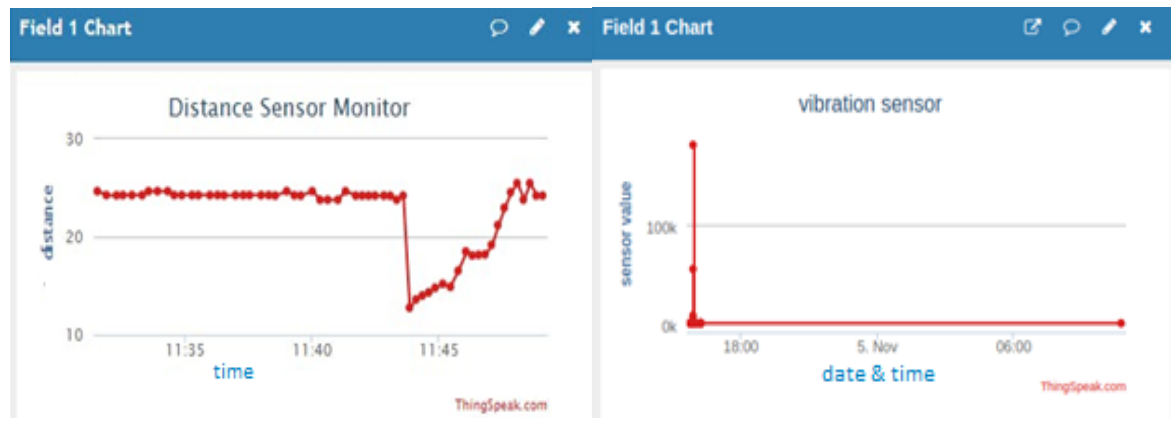

Figure 6.Distance measured between vehicle 1 Figure 7. Vibration measured between vehicle 1 and and 2 with respect to time. 2 with respect to date and time.

The Fig. 6shows the distance with respect to the date of the travel. If the distance between the vehicles varies then the graph also varies accordingly. The distance range between the vehicles in the setup is less than or equal to $30 \mathrm{~cm}$. The Fig. 7 shows relation between the sensor values with respect to the date \& time of the vehicle. When an accident takes places, the graph increases drastically. The vibration sensor measures the force caused during the collision of the vehicle.

\section{Conclusion}

This paper integrates the advantages of Li-Fi, Wi-Fi and the Black Boxsystems into one and so, increases the fault tolerance rate when either oneof the systems fail. The setup can be used as a proof of concept that at these experimental values (from basic components used in setup)and the alert messages are relayed correctly and accidentscan be prevented experimentally and theoretically. The system successfully uses visible light to transmit the data from one vehicle toanother. The data is stored in real-time in a cloud server with date and time and a SD card. The data uploaded to the cloud is easily accessible with legal authority and is also password protected to prevent any unauthorized access to the data thus making it secure.

\section{References}

[1] Nikhil Kumar, DebopamAcharya, and DivyaLohani, An IOT Based Vehicle Accident Detection and Clarification System using Sensor Fusion,

[2] Ali Hassan, M. Shahroz Abbas, MuhammedAsif, Maaz Bin Ahmad, M. Zubair Tariq, An Automatic Accident Detection System: A Hybrid Solution, $20194^{\text {th }}$ International Conference on Information Systems Engineering (ICISE).

[3] Rahul George, SrikumarVaidyananthan, Amandeep Singh Rajput, K Deepa, LiFi for Vehicle to Vehicle Communication - A Review, International conference on recent trends in advanced computing 2019, ICRTAC 2019.

[4] HN. H. Sane, D. S. Patil, S. D. Thakare, and A. V. Rokade, Real Time Vehicle Accident Detection and Tracking Using GPS and GSM , International Journal on Recent and Innovation Trends in Computing and Communication, Archived from the Original, Accessed 24 June 2018.

[5] V. Anupriya, B. Lissy Roy, V. Dheepthi and F. Masood. Smart Accident Notification and Collision Avoidance System . International Journal of Engineering Research and Technology, 4(4), pp. 1148$1152,2015$.

[6] Hema Patel, Survey on Li-Fi Technology and Its Application . International Journal of Information Sciences and Techniques (IJIST) Vol .6, No.1/2, March 2016. 